\title{
Retention of participants recruited to a multi-year longitudinal study via Prolific
}

\author{
Emily J. Kothe ${ }^{1} \&$ Mathew Ling ${ }^{1}$ \\ ${ }^{1}$ Deakin University \\ Geelong \\ Australia \\ Misinformation Lab \\ School of Psychology
}

\begin{abstract}
Despite increasing use of the crowdsourcing platform Prolific in psychological research, there is limited data on the retention of participants in longitudinal studies using this recruitment channel. We present a summary of retention within a study on vaccination attitudes with three time points conducted via Prolific. Of the 331 participants who completed the initial survey in 2018, 254 (76.74\% of total Time 1 sample; $96.95 \%$ of active users) completed the Time 2 survey approximately 12 months later, and 205 (61.93\% of total Time 1 sample) completed the Time 3 survey at 19 months. At both Time 2 and Time 3 the majority of participants completed the follow-up survey within 24 hours of survey launch. Older participants were more likely to be retained. However, retention was not related to gender, educational attainment, health related belief or behaviour, speed of survey completion, or time to recruitment at Time 1. Overall, Prolific shows similar retention characteristics to conventional cohort studies with substantially reduced researcher burden.
\end{abstract}

Keywords: Longitudinal, Prolific, online research, attrition

Word count:

Online crowdsourcing platforms are increasingly common in research, with over 15,000 papers referencing Amazon's popular Mechanical Turk (MTurk) platform published between 2006 and 2014 (Chandler \& Shapiro, 2016) and the use of Amazon MTurk increasing in marketing and consumer behaviour (Goodman \& Paolacci, 2017; Hulland \& Miller, 2018), cognitive psychology (Stewart et al., 2017), addiction (Strickland \& Stoops, 2019), organisational psychology (Behrend et al., 2011) and personality and social psychology (Zhou \& Fishbach, 2016). The rapid development of this as a method for efficiently recruiting a large sample of participants who may be more attentive (Hauser \& Schwarz, 2016) than student samples or belong to hard to reach populations (Smith et al., 2015) has fuelled the development of researchfocussed platforms like Crowdflower and Prolific (Palan \& Schitter, 2018).

Peer et al. (2017) conducted a comparison of MTurk and Prolific across two studies. They concluded that Prolific data quality was comparable to MTurk across multiple metrics and noted that participants from Prolific reported higher levels of naivety, spent less time on the platform, and were more demographically diverse. Given evidence from other studies that frequent exposure to the same or similar experimental paradigms from "superworkers" on the MTurk platform may reduce reported effect sizes and difficulty in replicating previously observed effects (see Chandler et al., 2019), the relative naivety of the Prolific subject pool makes Prolific an enticing alternative to MTurk.

As Prolific is specifically designed for use in the research context, the developers of the platform have made specific efforts to address the needs of researchers, for example providing estimates of the available population, pre-screening and exclusion of participants, as well as whitelisting, permitting the collection of longitudinal data (Palan \& Schitter, 2018). However, there is little data available on the retention of participants in longitudinal studies conducted on the platform. The instructions for setting up a longitudinal study on Prolific note that "We've had studies with $0 \%$ dropout rate and as high as $50 \%$. A typical study would be somewhere in between these extremes" (Moodie, 2018) but does not specify the timescales over which such studies have measured attrition. Palan and Schitter (2018) report a study in a two-week follow-up that experienced $23.75 \%$ attrition when recruiting inexperienced Prolific users and $6.88 \%$ attrition when recruiting users with high experience. The Prolific blog provides an account of a "longitudinal" study with no attrition, however that study included pre-test assessment in Week 1, intervention phase in Weeks 2-5, and post-test in 
Week 6 (Lettemann \& Damer, 2018). Lee et al. (2020) conducted three waves of data collection via Prolific in March and April of 2020, attrition between Wave 1 to Wave 3 was $16.7 \%$.

Sheeran and Conner (2019) reported a three-month followup that involved completion of two questionnaires threemonths apart with $70 \%$ retention between the two timepoints. This provides a somewhat more informative test of retention that the very short follow-up periods described above. However, the authors did not compare the characteristics of those that completed to those who completed only one, or describe the methodology in sufficient detail to allow for calculation of the effective rate of pay. Given that conditional attrition of participants might result in the induction of an illusory timeseries effect and that rate of pay may influence retention to longitudinal studies (Auer et al., 2021) it seems pertinent to determine, where possible, any correlates of retention over time.

To support future planning of longitudinal research, we provide a summary of our attrition statistics from a study examining beliefs and behaviours regarding vaccination behaviour with three time points. Time 1 and Time 2 were separated by approximately 12 months (331-377 days). The Time 1 and Time 3 were separated by approximately 19 months (952 1008 days) meaning that the time between Time 2 and Time 3 was 578-673 days.

\section{Methods}

We report how we determined our sample size, all data exclusions (if any), all manipulations, and all measures in the study.

\section{Participants}

In order to be eligible Prolific users needed to be residents of the UK and aged 18 years or older at the time of Time 1 recruitment. The intention was to recruit 330 participants to Time 1, however 332 provided complete data at that timepoint. It appears that a small amount of oversampling occurred when participants did not enter their completion code into the Prolific platform immediately after completion of the study. This resulted in Prolific marking those responses as timed out and automatically recruiting additional participants to the Time 1 sample. The sample size at baseline was determined on the basis of the available budget at that timepoint.

\section{Materials and Procedure}

The measures in each wave of this study are available on the OSF [https://osf.io/yjstk/].
Time 1

At Time 1, the study was advertised on the Prolific platform using the following text:

"In this survey, you will be asked questions about your demographic characteristics (e.g. age and gender) and your beliefs and behaviours regarding seasonal influenza vaccinations.

Participation in the study involves completion of two surveys, one now and one at the end of the 2018-2019 flu season (late May 2019). Each survey will take approximately 10 minutes and you will be reimbursed $£ 1.30$ for each survey that you choose to complete.

\section{If you choose to take part in the survey now and then decide not to take part in the follow- up survey in 2019 you will still receive reim- bursement for the time and effort involved in the first survey."}

Age and nationality pre-screens were applied to ensure that participants were aged $18+$ and had previously identified their nationality as "United Kingdom."

Upon advancing to the survey proper, participants were asked to provide age, gender, and highest level of education before providing data on their willingness and intention to vaccinate, past vaccination behaviour, beliefs and attitudes towards seasonal influenza vaccination. Specifically, consistent with Protection Motivation Theory (Rogers, 1975), participants were asked about (a) their susceptibility to infection, (b) the severity if infected, (c) any benefits from contracting influenza, (d) their ability to obtain a vaccine, (e) the efficacy of the vaccine in preventing infection, and (f) the severity of any costs associated with obtaining the vaccine. Consistent with the Prototype Willingness Model (Gibbons \& Gerrard, 1995), participants also provided responses on (a) the favourability of their impression of someone who got the vaccine annually, (b) their willingness to receive the vaccine if presented with an opportunity, (c) their attitude towards receiving the vaccine, and (d) their perception of the injunctive norm to receive the vaccine.

\section{Time 2 (1 year follow-up)}

At Time 2 the study was advertised to participants who had completed the Time 1 study using a custom whitelist as per the instructions provided in (Moodie, 2018). The study was advertised on the Prolific platform using the following text: 
"In this survey, you will be asked questions about your beliefs and behaviours regarding seasonal influenza vaccinations.

This is a follow-up to a study you completed in 2018. Taking part in this survey will take approximately 10 minutes and you will be reimbursed £1.30 for completion.”

Upon advancing to the survey proper, participants responded to the same set of items measuring vaccination-related beliefs and behaviour they had completed at Time 1. In addition they completed a measure of general vaccine hesitancy. They did not provide demographic characteristics in the Time 2 survey.

\section{Time 3 (19 month follow-up)}

At Time 3 the study was advertised to participants who had completed the Time 1 study using a custom whitelist as per the instructions provided in (Moodie, 2018). The recruitment strategy allowed for the recovery of participants who completed Time 1 but were missing from Time 2 . The study was advertised on the Prolific platform using the following text:

"In this survey, you will be asked questions about your beliefs and behaviours regarding COVID-19 vaccinations.

This is a follow-up to a study you completed about seasonal influenza vaccinations in 2018 and 2019. Taking part in this survey will take approximately 10 minutes and you will be reimbursed £1.30 for completion."

Upon advancing to the survey proper, participants responded to a similar set of items as they had completed at the earlier timepoints. However, rather than measuring beliefs about influenza vaccinations the items had been adapted to measure beliefs about COVID-19 vaccination. Participants also provided information about their exposure to COVID-19 and their COVID-19 vaccination status.

\section{Data analysis}

We used R [Version 4.0.3; R Core Team (2018)] and the Rpackages dplyr [Version 1.0.5; Wickham et al. (2019)], ggplot2 [Version 3.3.3; Wickham (2016)], here [Version 1.0.1; Müller (2017)], papaja [Version 0.1.0.9997; Aust and Barth (2018)], and tableone [Version 0.12.0; Yoshida and Bohn. (2018)] for all our analyses.

\section{Results}

\section{Speed of recruitment and overall retention rates}

Time 1 recruitment commenced at 2018-07-25 07:43:10 UTC and concluded at 2018-07-25 10:00:39 UTC. Time 1 recruitment of all 332 participants occurred over 2.29 hours. The pace of recruitment over time is shown in Figure 1, in this figure each dot represents a response from a single participant while the solid black line shows the growth of the total sample over time. One participant was excluded at Time 1 because they indicated that they were not a UK resident. A single participant indicated an implausible value for age (age $=3$ years), the age data for this participant was re-coded as missing but all other data was retained for analysis. As a result, when calculating retention, the denominator for all percentages is 331 .

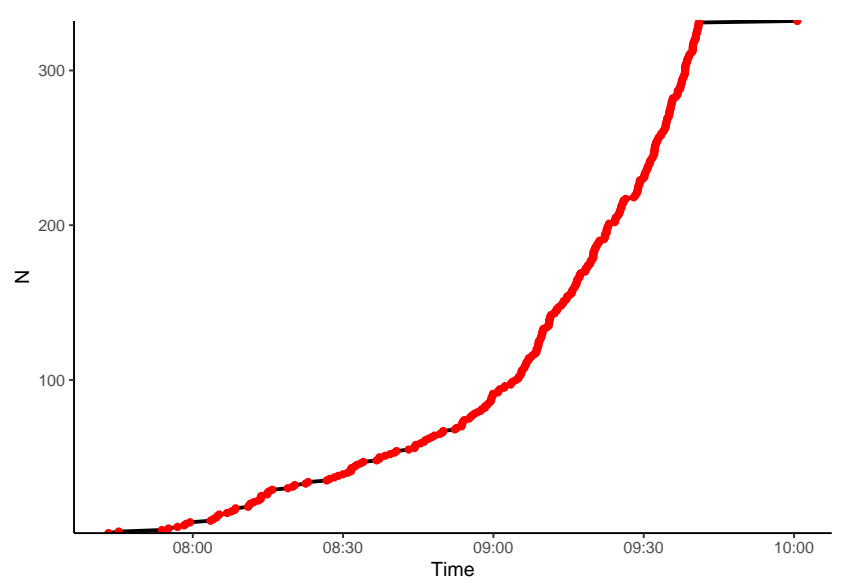

Figure 1

Recruitment over time for Time 1 sample

At Time 2, 262 participants from the Time 1 sample had been active on Prolific in the last 90 days (79.15\%). Time 2 recruitment commenced at 2019-06-21 00:35:52 UTC and concluded at 2019-08-06 19:10:07 UTC (46.77 days). In this time 254 participants completed the Time 2 survey $(76.74 \%$ of total Time 1 sample; $96.95 \%$ of active users at start of Time 2. The pace of recruitment over time is shown in Figure 2 , in this figure each dot represents a response from a single participant while the solid black line shows the growth of the total sample over time.

Time 3 recruitment commenced at 2021-03-02 22:32:47 UTC and concluded at 2021-04-28 07:33:21 UTC (56.38 days). In this time 205 participants completed the Time 3 survey (61.93\% of total Time 1 sample). Unfortunately, a change in the Prolific interface between Time 2 and Time 3 means that it is not possible to determine the number of participants who had been active on Prolific in the last 90 days 


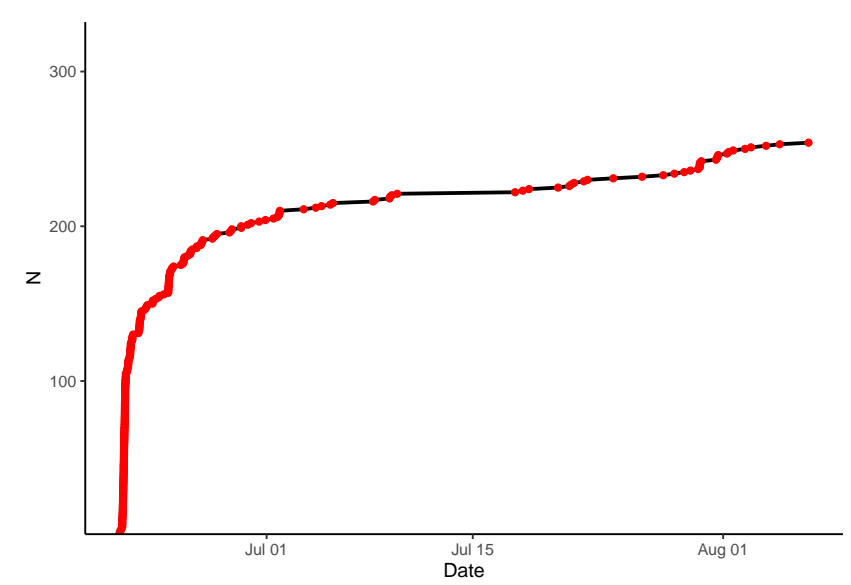

Figure 2

Recruitment over time for Time 2 sample

when Time 3 commenced. The pace of recruitment over time is shown in Figure 3, in this figure each dot represents a response from a single participant while the solid black line shows the growth of the total sample over time. It should be noted that respondents at Time 3 included 22 who did not respond at Time 2. Given missingness is a regular feature of longitudinal studies we have reported the recovered counts at each time to reflect an estimate of the recoverable sample, however there are only 183 complete cases over the life of the study.

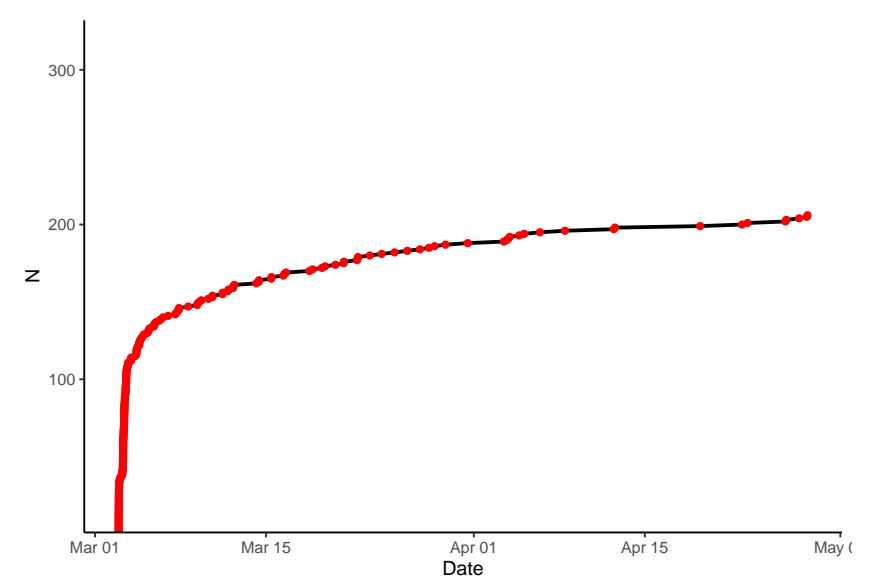

Figure 3

Recruitment over time for Time 3 sample

\section{Comparison of demographic characteristics stratified by the last timepoint completed}

The age, gender, and level of highest educational attainment was collected from participants at Time 1. Participants were grouped according to the last time point where they provided and compared on the basis of these characteristics (see Table 1). According to an ANOVA, age was related to attrition. A post-hoc Tukey HSD test was conducted to examine this effect. There was a significant difference in age between those who last provided data Time 1 and Time $3(p<.001)$ and between those who last provided data at Time 2 and Time $3(p=.012)$. The difference in age between those who last provided data at Time 1 and Time 2 was not significant. Chisquared tests indicated no systematic differences in gender or educational attainment between people who last provided data at Time 1, Time 2, or Time 3.

\section{Comparison of vaccination related beliefs and behaviour stratified by the last timepoint completed}

The purpose of this longitudinal study was to examine the role of beliefs about influenza vaccination on subsequent vaccination uptake. Systematic attrition could causes misestimation in the final analyses. As such, differential attrition was examined using a series of ANOVA analyses (see Table 2 ). There were no differences observed on any category of Time 1 beliefs on the basis of the last timepoint the participant completed.

To assess for differential attrition on the basis of prior vaccination status, the relationship between the last timepoint at which a participant provided data and having received an influenza vaccine in 2017-2018 (i.e. the influenza season prior to the study) was evaluated. There was no difference in 2017-2018 vaccination status between participants who last provided data at Time 1 (23.21\% vaccinated in 2017 2018), Time 2 (25.35\% vaccinated in 2017-2018) and Time $3(29.76 \%$ vaccinated in $2017-2018)\left(\chi^{2}(2, n=331)=1.19\right.$, $p=.551)$.

\section{Comparison of other characteristics stratified by the last timepoint completed}

The length of time each participant took to complete the Time 1 survey was automatically recorded by the survey software. ANOVA indicated no differences in survey completion time on the basis of the last timepoint the participant completed ( $\mu$ Time 1: $517.98, \mu$ Time 2: 399.82 seconds; $\mu$ Time 3: 384.65 seconds; $F(2,329)=1.55, M S E=255,338.95$, $p=.214, \hat{\eta}_{G}^{2}=.009$ ).

The length of time between study launch when each participant commenced Time 1 survey completion was calculated. ANOVA indicated no differences in time to recruitment on the basis of the last timepoint the participant completed ( $\mu$ Time 1: 80.95 minutes, $\mu$ Time 2: 88.64 minutes, $\mu$ Time 3: 88.06 minutes; $F(2,329)=1.59, M S E=2,796,134.91$, $\left.p=.206, \hat{\eta}_{G}^{2}=.010\right)$. 
RETENTION OF PARTICIPANTS

Table 1

Demographic characteristics of participants stratified by the last timepoint completed

\begin{tabular}{lcccc}
\hline & Time 1 & Time 2 & Time 3 & Test Statistics \\
\hline $\mathrm{n}$ & 56 & 71 & 205 & \\
Age at Time 1 (mean (SD)) & $32.57(10.40)$ & $34.04(9.89)$ & $38.95(13.74)$ & $F(2,328)=7.96, M S E=155.78, p<.001$ \\
Gender = Male (\%) & $19(33.9)$ & $16(22.5)$ & $48(23.4)$ & $\chi^{2}(2, n=331)=2.89, p=.236$ \\
Education at Time 1 (\%) & & & $\chi^{2}(10, n=331)=8.40, p=.590$ \\
No formal qualifications & $0(0.0)$ & $1(1.4)$ & $2(1.0)$ & $32(15.6)$ \\
Secondary school/GCSE & $7(12.5)$ & $11(15.5)$ & $64(31.2)$ & \\
College/A levels & $19(33.9)$ & $29(40.8)$ & $70(34.1)$ & \\
Undergraduate degree (BA/BSc/other) & $24(42.9)$ & $23(32.4)$ & $7(9.9)$ & $5(2.4)$ \\
Graduate degree (MA/MSc/MPhil/other) & $6(10.7)$ & $7(9.6)$ & \\
Doctorate degree (PhD/MD/other) & $0(0.0)$ & $0(0.0)$ & & \\
\hline
\end{tabular}

Table 2

Beliefs about influenza stratified by the last timepoint completed

\begin{tabular}{lcccc}
\hline Construct & Time 1 Mean $(S D)$ & Time 2 Mean $(S D)$ & Time 3 Mean $(S D)$ & $p$ \\
\hline $\mathrm{n}$ & 56 & 71 & 205 & \\
Severity & $3.61(0.83)$ & $3.70(0.85)$ & $3.61(0.90)$ & 0.738 \\
Susceptibility & $3.32(0.87)$ & $3.34(1.07)$ & $3.30(0.93)$ & 0.952 \\
Response Costs & $2.47(0.73)$ & $2.38(0.89)$ & $2.27(0.75)$ & 0.184 \\
Self-Efficacy & $4.12(0.80)$ & $4.17(0.81)$ & $4.31(0.80)$ & 0.202 \\
Response Efficacy & $3.27(0.98)$ & $3.12(1.04)$ & $3.09(0.84)$ & 0.418 \\
MRR & $2.97(0.73)$ & $2.92(0.99)$ & $3.03(0.88)$ & 0.646 \\
Prototype & $1.61(1.80)$ & $0.99(1.70)$ & $1.27(1.91)$ & 0.172 \\
Willingness & $3.46(1.23)$ & $3.37(1.19)$ & $3.37(1.22)$ & 0.900 \\
Attitude & $3.84(0.82)$ & $3.80(1.05)$ & $3.93(0.94)$ & 0.540 \\
Subjective Norm & $3.88(0.76)$ & $3.83(0.90)$ & $3.77(0.87)$ & 0.703 \\
Intention & $2.94(1.45)$ & $2.83(1.49)$ & $2.83(1.55)$ & 0.886 \\
\hline
\end{tabular}

Note. MRR = Maladaptive Response Rewards

\section{Discussion}

Meta-analysis indicates that average retention to longitudinal cohort studies is $73.5 \%$ (Teague et al., 2018). The retention observed within this study $(76.74 \%)$ at 1 year and $(61.93 \%)$ at 19 months is broadly consistent with this average and compares favourability to retention observed by Palan and Schitter (2018) and Sheeran and Conner (2019) in other studies conducted on the Prolific platform with shorter follow-up periods. Interestingly, retention was higher than that reported for longitudinal studies conducted on MTurk (Chandler et al., 2014; Christenson \& Glick, 2013). Chandler et al. (2014) reported that only $44 \%$ of MTurk respondents completed a follow-up survey one year later. While Christenson and Glick (2013) reported that $63 \%$ of participants were retained after 4 months in a 5 wave MTurk panel study. The high retention rate in this study and in others that have used crowdsourcing platforms for data collection is particularly promising given that very low burden to researchers in managing participant retention and recontact. While we did not formally track researcher time, we estimate that management each wave of follow-up recruitment process required less than three hours of researcher time at that timepoint (including the set-up of Prolific study details at follow-up, management of the custom whitelist, and participant payment).

Retention was selective on the basis of age, but not gender, educational attainment, or health related beliefs and behaviour. A number of longitudinal cohort studies of adults have previously reported greater attrition among younger participants (e.g. Young et al., 2006). As such, the extent 
that selective attrition occurs, it mirrors that likely to be observed when using other methods of recruitment. Selective attrition on the basis of gender is common in cohort studies. Teague et al. (2018) reported higher retention rates in cohorts with a greater proportion of female participants and examinations within individual cohort studies have often reported higher attrition among male respondents (e.g. Radler \& Ryff, 2010). Differential attrition on the basis of gender was not observed here which may indicate an advantage of this recruitment channel for retaining male participants.

Furthermore, recovery of participants within Time 2 was non-linear with $51.18 \%$ of the Time 2 sample recovered within 24 hours, 58.66\% in 48, and 61.42\% in 72 (see Figure 2). Recovery followed a similar pattern within Time 3, $54.15 \%$, of the Time 3 sample recovered within 24 hours, $61.95 \%$ in 48 , and $66.34 \%$ in 72 (see Figure 3). This may permit researchers to limit the length of their follow-up period and still recover a majority of participants to within a given wave. However, older participants were recovered more rapidly than younger participants at both Time $2(r=$ $-.15,95 \% \mathrm{CI}[-.26,-.02], t(251)=-2.32, p=.021)$ and Time $3(r=-.31,95 \%$ CI $[-.42,-.18], t(203)=-4.57$, $p<.001$ ). As such, limiting the length of time that a survey is available may exacerbate selective attrition related to age.

Caution should be taken in extrapolating from this data, particularly to other crowdsourcing platforms. While there are constraints on participant incentive within Prolific that increase the consistency of experience of participants across studies to some extent, it is likely that the content and length of the studies may affect retention. Furthermore, unmeasured variables that may be pertinent to future research questions are likely to have influenced retention. For example, the rate of pay $£ 1.30$ per survey is likely to have influenced retention, especially since the majority of participants completed the Time 1 survey in less than the advertised expected time. Studies with higher or lower rates of pay may experience lower attrition (see Auer et al., 2021). However, given that the majority of loss to follow-up occurred when participants were no longer on the platform at all, this effect may be overshadowed by the broader factors that cause Prolific users to become inactive. Interestingly, the rate of inactivity is much lower than has been estimated for MTurk, where capturerecapture techniques have estimated that every three months $26 \%$ of MTurk workers retire from the platform (Stewart et al., 2015).

Given that contacting participants outside of Prolific is a breach of the terms of service, many strategies shown to be effective in increasing retention in conventional cohort designs (Booker et al., 2011; e.g. Teague et al., 2018) are not viable in this context. Consequently, the proportion of active users on the platform at a follow-up timepoint provides an upper limit of a study's retention rate. Predictors of recov- ery of this maximum will need to be explored independently, through meta-analysis of retention statistics in future longitudinal studies on this platform.

This study provides the first comprehensive analysis of retention to a longitudinal study conducted on the Prolific. The findings suggest that Prolific is a feasible and cost effective method of recruiting to such studies. However, more reports of longitudinal studies conducted using the platform will be required in order to allow researchers to predict likely retention to studies using other designs and increased understanding of the factors that influence retention both to the platform and to individual studies. 


\section{References}

Auer, E. M., Behrend, T. S., Collmus, A. B., Landers, R. N., \& Miles, A. F. (2021). Pay for performance, satisfaction and retention in longitudinal crowdsourced research. Plos One, 16(1), e0245460.

Aust, F., \& Barth, M. (2018). papaja: Create APA manuscripts with R Markdown. https://github.com/crsh/ papaja

Behrend, T. S., Sharek, D. J., Meade, A. W., \& Wiebe, E. N. (2011). The viability of crowdsourcing for survey research. Behavior Research Methods, 43(3), 800-813.

Booker, C. L., Harding, S., \& Benzeval, M. (2011). A systematic review of the effect of retention methods in population-based cohort studies. BMC Public Health, 11(1), 249.

Chandler, J., Mueller, P., \& Paolacci, G. (2014). Nivete among amazon mechanical turk workers: Consequences and solutions for behavioral researchers. Behavior Research Methods, 46(1), 112-130.

Chandler, J., Rosenzweig, C., Moss, A. J., Robinson, J., \& Litman, L. (2019). Online panels in social science research: Expanding sampling methods beyond mechanical turk. Behavior Research Methods, 51(5), 2022-2038.

Chandler, J., \& Shapiro, D. (2016). Conducting clinical research using crowdsourced convenience samples. Annual Review of Clinical Psychology, 12.

Christenson, D. P., \& Glick, D. M. (2013). Crowdsourcing panel studies and real-time experiments in MTurk. The Political Methodologist, 20(2), 27-32.

Gibbons, F. X., \& Gerrard, M. (1995). Predicting young adults' health risk behavior. Journal of Personality and Social Psychology, 69(3), 505.

Goodman, J. K., \& Paolacci, G. (2017). Crowdsourcing consumer research. Journal of Consumer Research, 44(1), 196-210.

Hauser, D. J., \& Schwarz, N. (2016). Attentive Turkers: MTurk participants perform better on online attention checks than do subject pool participants. Behavior Research Methods, 48(1), 400-407.

Hulland, J., \& Miller, J. (2018). “Keep on Turkin’?” Springer.

Lee, S. J., Ward, K. P., \& Rodriguez, C. (2020). Longitudinal analysis of short-term changes in relationship conflict during COVID-19: A risk and resilience perspective. PsyArXiv. https://doi.org/10.31234/osf.io/yqv7j

Lettemann, H., \& Damer, E. (2018). An honest review: Two researchers share their thoughts on running a complex longitudinal study via Prolific. In Prolific Blog. https://blog.prolific.co/an-honest-review-tworesearchers-share-their-\%20thoughts-on-running-acomplex-longitudinal-study-via-prolific/

Moodie, J. (2018). Minimising dropout / attrition rate in longitudinal studies. In Prolific Help Centre. https://researcher-help.prolific.co/hc/engb/articles/360009223613-Minimising-dropoutattrition-rate-in-longitudinal-studies

Müller, K. (2017). Here: A simpler way to find your files. https://CRAN.R-project.org/package=here

Palan, S., \& Schitter, C. (2018). Prolific. Ac-A subject pool for online experiments. Journal of Behavioral and Experimental Finance, 17, 22-27.

Peer, E., Brandimarte, L., Samat, S., \& Acquisti, A. (2017). Beyond the turk: Alternative platforms for crowdsourcing behavioral research. Journal of Experimental Social Psychology, 70, 153-163.

R Core Team. (2018). R: A language and environment for statistical computing. R Foundation for Statistical Computing. https://www.R-project.org/

Radler, B. T., \& Ryff, C. D. (2010). Who participates? Accounting for longitudinal retention in the MIDUS national study of health and well-being. Journal of $A g$ ing and Health, 22(3), 307-331. https://doi.org/10.1177/ 0898264309358617

Rogers, R. W. (1975). A protection motivation theory of fear appeals and attitude Change1. The Journal Of Psychology, 91(1), 93-114.

Sheeran, P., \& Conner, M. (2019). Degree of reasoned action predicts increased intentional control and reduced habitual control over health behaviors. Social Science \& Medicine, 228, 68-74.

Smith, N. A., Sabat, I. E., Martinez, L. R., Weaver, K., \& $\mathrm{Xu}, \mathrm{S}$. (2015). A convenient solution: Using MTurk to sample from hard-to-reach populations. Industrial and Organizational Psychology, 8(2), 220-228.

Stewart, N., Chandler, J., \& Paolacci, G. (2017). Crowdsourcing samples in cognitive science. Trends in Cognitive Sciences, 21(10), 736-748.

Stewart, N., Ungemach, C., Harris, A. J., Bartels, D. M., Newell, B. R., Paolacci, G., Chandler, J., \& others. (2015). The average laboratory samples a population of 7,300 amazon mechanical turk workers. Judgment and Decision Making, 10(5), 479-491.

Strickland, J. C., \& Stoops, W. W. (2019). The use of crowdsourcing in addiction science research: Amazon Mechan- 
ical Turk. Experimental and Clinical Psychopharmacology, 27(1), 1 .

Teague, S., Youssef, G. J., Macdonald, J. A., Sciberras, E., Shatte, A., Fuller-Tyszkiewicz, M., Greenwood, C., McIntosh, J., Olsson, C. A., \& Hutchinson, D. (2018). Retention strategies in longitudinal cohort studies: A systematic review and meta-analysis. BMC Medical Research Methodology, 18(1), 151.

Wickham, H. (2016). ggplot2: Elegant graphics for data analysis. Springer-Verlag New York. http://ggplot2.org

Wickham, H., François, R., Henry, L., \& Müller, K. (2019). Dplyr: A grammar of data manipulation. https://CRAN. R-project.org/package $=$ dplyr

Yoshida, K., \& Bohn., J. (2018). Tableone: Create 'table 1 ' to describe baseline characteristics. https://CRAN.Rproject.org/package $=$ tableone

Young, A. F., Powers, J. R., \& Bell, S. L. (2006). Attrition in longitudinal studies: Who do you lose? Australian and New Zealand Journal of Public Health, 30(4), 353-361.

Zhou, H., \& Fishbach, A. (2016). The pitfall of experimenting on the web: How unattended selective attrition leads to surprising (yet false) research conclusions. Journal of Personality and Social Psychology, 111(4), 493.

\section{Contributions}

Contributed to conception and design: EK \& ML. Contributed to acquisition of data: EK \& ML. Contributed to analysis and interpretation of data: EK \& ML. Drafted and/or revised the article: EK \& ML. Approved the submitted version for publication: EK \& ML.

\section{Acknowledgements}

The authors wish to acknowledge the contribution of the individual participants to this research. Without their contributions the research would quite literally not exist.

\section{Funding information}

This project was funded by funds provided to Emily Kothe by the School of Psychology at Deakin University.

\section{Competing interests}

No competing interests exist.

\section{Data accessibility statement (mandatory)}

All data, materials, and analytic code for this project can be found on the OSF [https://osf.io/yjstk/].

\section{Figure titles and legends}

- Figure 1. Recruitment over time for Time 1 sample

- Figure 2. Recruitment over time for Time 2 sample

- Figure 3. Recruitment over time for Time 3 sample 